\title{
Tourist spending dynamics in the Algarve: a cross-sectional analysis \\ JAIME SERRA
}

Interdisciplinary Centre for History, Culture and Societies (CIDEHUS.UE), and School of

Social Sciences, Universidade de Évora, Largo dos Colegiais 2, 7004-516 Évora, Portugal.

E-mail: jserra@uevora.pt. ANTÓNIA CORREIA

Center for Advanced Studies in Management and Economics (CEFAGE-UE), and Faculty

of Economics, Universidade do Algarve, Campus das Gambelas, 8005-139 Faro, Portugal.

E-mail: acorreia@ualg.pt.

PAULO M.M. RODRIGUES

Banco de Portugal, Nova School of Business and Economics, Universidade Nova de Lisboa,

Campus de Campolide, 1099-032 Campolide, Lisbon, Portugal. E-mail:

prodrig@novasbe.pt.

This paper assesses the determinants of international tourists' spending in the Algarve from 2007 to 2010. Based on a sample of 15,542 observations a cross-section model was estimated using ordinary least squares. The results reveal that a combination of socio-demographic, behavioural and motivation variables explain the spending patterns of international tourists in the Algarve. Analysis of the data indicates that tourist motivations related to accommodation facilities, cultural and historical resources, gastronomy, hospitality, prices and sightseeing tours positively affect tourists' spending. Moreover, the findings provide evidence that, over the years, the most loyal markets in Algarve reveal changing patterns in their spending behaviour. Destination management authorities should be aware of this spending behaviour of second-generation sun and sand visitors and should formulate strategies accordingly. Policy and managerial recommendations are discussed.

Keywords: tourism demand; tourist spending; travel motivations; microeconomic analysis; multiple regression analysis; Algarve 